\title{
A Fitness-Independent Evolvability Measure for Evolutionary Developmental Systems
}

\author{
Yaochu Jin, Senior Member, IEEE, and Jens Trommler
}

\begin{abstract}
Evolvability refers to the organisms ability to create heritable new phenotypes that potentially facilitate the organism's survival and reproduction. In this paper, a general evolvability measure for a computational model of evolutionary development is proposed. The measure is able to quantify individuals' evolvability, including robustness and innovation, independent of the fitness function of the evolutionary system. Empirical studies are performed to check the evolvability of individuals in in silico evolution of oscillatory behavior using the proposed evolvability measure. Our preliminary results suggest that evolvability of the developmental system can evolve without an explicit selection pressure on evolvability, confirming findings revealed in other artificial evolutionary systems.
\end{abstract}

\section{INTRODUCTION}

Evolvability is an important concept both in biology and evolutionary computation. In biology, one widely known definition for evolvability was given by Wagner [1]. According to Wagner, a biological system is evolvable if its properties show heritable genetic variation and if it can acquire novel functions through genetic changes, which should help the organism to survive and reproduce. Another definition for evolvability was suggested by Barnett [2], which considers a variational system's evolvability as the probability that an offspring has a better fitness than its parent. A similar definition was also proposed by Turney [3], which believes that individual $\mathrm{A}$ is more evolvable than individual $\mathrm{B}$ if the best offspring of $\mathrm{A}$ is on average fitter than the best offspring of $\mathrm{B}$.

Evolvability defined in [2], [3] is dependent on the fitness function. However, a fitness-dependent evolvability measure for evolutionary systems may be of less interest since it is problem-specific and not well suited for systems in a changing environment. To address this problem, other evolvability definitions that are less dependent on fitness of the evolutionary systems have also been proposed.

Reisinger et al [4] claimed that evolvability can be seen as the variance of the efficiency of evolution (the average of the best fitness over the whole evaluation phase) over all drift rates. The authors have also compared evolvability of direct and indirect coding and their results indicated that evolvability of direct coding is lower than that of indirect coding like gene regulatory networks. Izquierdo and Fernando [5] discussed the evolvability of a gene transcription network model using hierarchical metagraphs. The evolvability of such graphs is then defined as the the navigability in phenotype space in response to mutations in the transcription factor binding sequence.

Yaochu Jin is with the Honda Research Institute Europe, Carl-Legien-Str. 30, 63073 Offenbach, Germany (Email: yaochu.jin@honda-ri.de).

Jens Trommler is with the Institut für Theorie Elektromagnetischer Felder, Technische Universität Darmstadt, 64289 Drmstadt, Germany (Email: trommler@temf.tu-darmstadt.de).
Ciliberti et al [6] examined the evolvability of a discrete gene regulatory model in terms of the trade-off between innovation (phenotypic variability) and robustness. It was concluded that robustness may harm short-term innovation, but robustness is essential for the emergence of long-term innovation. Jin et al [7] investigated the relationship between innovation and robustness of a Boolean genotype-phenotype mapping. Innovation was defined as the average local variability (the probability of encountering a new phenotype in the presence of a genotypic mutation). The connection matrix of the mapping was then evolved using a multi-objective evolutionary algorithm. It was shown that there is an inherent trade-off between innovation and robustness.

This paper suggests an evolvability measure for an evolutionary developmental model for cell growth [8]. The developmental model is essentially a set of continuous nonlinear differential equations, whose phenotype is described by the state of the equations representing the concentration of functional proteins involved in cellular growth, including transcription factors. The proposed evolvability measure is independent of any specific fitness function and therefore is general for gene regulatory models based on differential equations. With the help of the proposed evolvability measure, the paper then examines the change of evolvability of the developmental model evolving for limit cycles. It is demonstrated that evolvability, albeit not under an explicit selection pressure on evolvability, increases steadily during the evolution, particularly when the mutation step-size is small.

\section{An Evolutionary Developmental Model for Cell Growth}

\section{A. Environment}

In the cell growth model, each cell, represented by a threedimensional sphere, contains a virtual DNA. Cells sit in a physical environment of bounded three-dimensional space in which they can divide, migrate and interact with each other. For the simplicity in simulating protein diffusion, the environment is divided into a number of cubes. In the simulation, the concentration of the proteins is defined on the vertices of the cubes and the concentration at an arbitrary point within the cube can be approximated by a linear approximation of those at the eight neighboring vertices.

\section{B. Genome Representation}

The virtual DNA, also known as a genome, consists of a number of genes, each again is composed of a number of structural units (SUs) and regulatory units (RUs). An SU defines cellular functions such as cell division and / or production 
of transcription factors (TFs), while a RU determines whether an SU will be activated, i.e., whether its encoded functions are expressed. Whether a TF can activate or repress a gene depends on the degree of match between the affinity value of a TF and that of a RU. Each SU has nine loci of real values, specifying the properties of the encoded cellular functions. Each RU consists of eight loci encoding the parameters of a sigmoid activation function, namely, $\theta, \sigma$, and $C$, and defining the affinity properties of the RU, including the affinity value $a^{R U}$ and affinity range $\epsilon$. The exact meaning of the parameters will be described in the next section. Fig. 1 (a) illustrates the structure of a genome, including the loci of a RU and an SU. An example of an SU for producing TFs is given in Fig. 1 (b). Note that some of the loci both in the SUs and RUs are not used in the current model, and that a valid gene should start with one or more RUs followed by one or more SUs. In other words, genes starting with an SU or ending with a RU will not be translated.

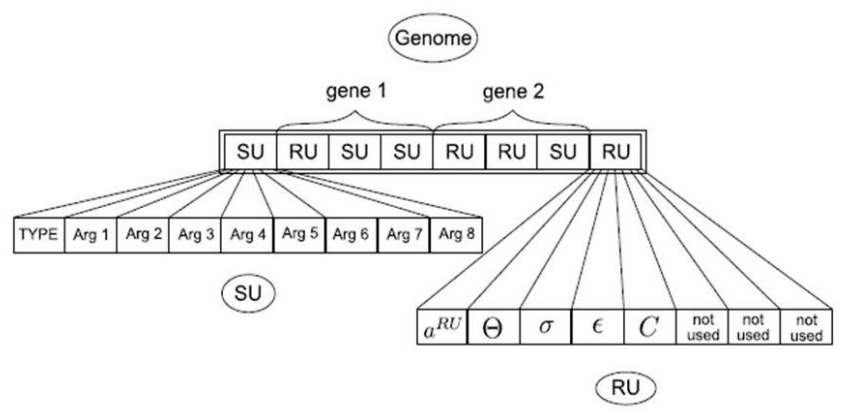

(a)

\begin{tabular}{|c|c|c|c|c|c|c|c|c|}
\hline $\begin{array}{c}\text { release } \\
\text { TF }\end{array}$ & $r$ & $\gamma$ & $D$ & $a^{T F}$ & $\begin{array}{c}\text { not } \\
\text { used }\end{array}$ & $e$ & $\begin{array}{c}\text { not } \\
\text { used }\end{array}$ & $\begin{array}{c}\text { not } \\
\text { used }\end{array}$ \\
\hline
\end{tabular}

(b)

Fig. 1. (a) Structure of a genome. (b) The loci in an SU encoding the cellular function of producing a transcription factor.

\section{Gene Regulatory Network}

Cellular growth of the studied system is under the control of a gene regulatory network (GRN) formed in the expression of genes in a virtual DNA illustrated in Fig. 1 (a). The activation level of an SU is determined by the sum of activity values of all its regulatory units $\left(\beta_{l}^{\mathrm{RU}}\right)$ :

$$
\beta_{l}^{\mathrm{gene}}=\sum_{l=1}^{n_{\mathrm{RU}}} \beta_{l}^{\mathrm{RU}}
$$

where $n_{\mathrm{RU}}$ is the number of the RUs of the $l$-th gene. If this activation level $\beta_{l}^{\text {gene }}$ is larger than zero, the gene is activated and its cellular functions are expressed.

The activity level of one RU is determined by the sum of the regulatory activities of all internal TFs (denoted by index $j$ ) and external TFs (denoted by index $i$ ):

$$
\begin{aligned}
\beta^{\mathrm{RU}}= & f_{\mathrm{nl}}^{\mathrm{RU}}\left(\sum_{i=1}^{n_{\mathrm{TF}}} f_{\mathrm{aff}}^{\mathrm{RU}}\left(d_{\mathrm{aff}, i}\right) \cdot c_{i}^{\mathrm{TF}}\right. \\
& \left.+\sum_{j=1}^{n_{\mathrm{TF}}} f_{\mathrm{aff}}^{\mathrm{RU}}\left(d_{\mathrm{aff}, j}\right) \cdot c_{j}^{\mathrm{TF}}\right),
\end{aligned}
$$

where $c_{i}^{\mathrm{TF}}$ and $c_{j}^{\mathrm{TF}}$ are the concentration of the external TFs and internal TFs, respectively, $d_{\text {aff }}$ is the degree of match between the affinity values of the RU and the TF:

$$
d_{\mathrm{aff}}=\min \left(\operatorname{abs}\left(a^{\mathrm{RU}}-a^{\mathrm{TF}}\right), 1-\operatorname{abs}\left(a^{\mathrm{RU}}-a^{\mathrm{TF}}\right)\right),
$$

and $f_{\text {aff }}^{\mathrm{RU}}\left(d_{\text {aff }}\right)$ is the affinity function:

$$
f_{\mathrm{aff}}^{\mathrm{RU}}\left(d_{\mathrm{aff}}\right)=e^{-0.5 \cdot\left(d_{\mathrm{aff}} / \epsilon\right)^{2}},
$$

and finally, $f_{\mathrm{nl}}^{\mathrm{RU}}(z)$ is a nonlinear activation function:

$$
f_{\mathrm{nl}}^{\mathrm{RU}}(z)=2 \cdot(2 \cdot \sigma-1)\left(\frac{1}{1+e^{(-30 \cdot C \cdot(z)-\Theta)}}-1\right) .
$$

In this regulatory model for cellular growth, the most important dynamics is that of the TFs in the environment. The TFs are produced by the SUs, the components of which are shown in Fig. 1. The type of the produced TF is identified with an affinity value $a^{\mathrm{TF}}$ (ID). The produced TF can be partly internal and partly external. The ratio between external and internal TFs is encoded by a parameter $e, 0 \leq e \leq 1$. The internal $\mathrm{TF}$ remains inside the cell and can regulate only the genes inside the cell, while the external TF can diffuse out of the cell and regulate the activation of genes of other cells. Index $i$ always denotes external TFs and index $j$ internal TFs. The production of a particular TF depends on the production rate $r$, the current activity of their corresponding genes $\beta_{m}^{\text {gene }}$ and the factor $e$. The Heaviside step function $H\left(\beta^{\text {gene }}\right)$ describes the activation function of genes. If $\beta^{\text {gene }}$ is higher than zero, $H\left(\beta^{\text {gene }}\right)$ is one and if it is below zero $H\left(\beta^{\text {gene }}\right)$ is zero. In case two different SUs have exactly the same affinity value $\left(a^{\mathrm{TF}}\right)$ in the genome, all parameters are averaged over the two SUs and then combined into one TF. Assume there are $n_{\text {gene }, i}$ genes that produce the same TF, the production of the TF can then be described by

$$
\begin{aligned}
& \frac{\partial \mathrm{TF}_{i}}{\partial t}=\sum_{m=1}^{n_{\text {gen }, i}} H\left(\beta_{m}^{\text {gene }}\right) \cdot r_{i} \cdot \beta_{m}^{\text {gene }} \cdot e_{i} \\
& \frac{\partial \mathrm{TF}_{j}}{\partial t}=\sum_{m=1}^{n_{\mathrm{gene}, j}} H\left(\beta_{m}^{\mathrm{gene}}\right) \cdot r_{j} \cdot \beta_{m}^{\mathrm{gene}} \cdot\left(1-e_{j}\right) \quad \text { internal TFs }
\end{aligned}
$$

Considering the decay of the produced proteins (with a decay rate $\gamma$ ), the concentration of internal TFs can be described as follows:

$$
\frac{\partial \mathrm{TF}_{j}}{\partial t}=\sum_{m=1}^{n_{\text {gene }, j}} H\left(\beta_{m}^{\text {gene }}\right) \cdot r_{j} \cdot \beta_{m}^{\text {gene }} \cdot\left(1-e_{j}\right)-\gamma_{j} \cdot \mathrm{TF}_{j} .
$$


The concentration of external TFs is influenced by both diffusion and decay, which can thus be described by

$$
\frac{\partial \mathrm{TF}_{i}}{\partial t}=\sum_{m=1}^{n_{\text {gene }, i}} H\left(\beta_{m}^{\mathrm{gene}}\right) \cdot r_{i} \cdot \beta_{m}^{\mathrm{gene}} \cdot e_{i}-\gamma_{i} \cdot \mathrm{TF}_{i}+D_{i} \cdot \nabla \mathrm{TF}_{i}
$$

As a whole, the dynamics of the GRN governing the cellular growth is described by a set of nonlinear differential equations.

\section{Genetic Variations}

An extended evolution strategy is employed to evolve the GRN dynamics. In this evolutionary algorithm, a population of $\mu$ individuals creates an offspring population of $\lambda$ individuals by changing the parents with Gaussian mutations. In addition to the mutation operator, other biologically plausible genetic operators, including gene duplication, deletion and transposition, are also introduced. With a given probability, one of the genetic operators, i.e., duplication, or deletion or transposition will be performed to the offspring produced by mutation. In gene duplication, a segment of genes in the genome is chosen randomly and then inserted into the genome at a random position. In gene transposition, a segment of randomly chosen genes is moved from the current position to another randomly chosen position. The deletion operator simply removes a randomly chosen gene segment.

\section{A Fitness-IndePEndent Evolvability MEASURE}

The primary goal of this work is to study the evolvability of the developmental model described in Section II regardless of a specific evolutionary task. As discussed in Section I, most existing evolvability measures are more or less fitnessdependent. Hence, it would be interesting to examine evolvability of developmental models independent of a problemspecific fitness function.

In addition, most existing evolvability measures are suggested for discrete direct or developmental representations [6], [5], [7]. In contrast, the development model studied in this work is more complex in that both genotypic and phenotypic spaces of the model are continuous, which have infinite number of states. The evolvability measure we are seeking has therefore to be able to cope with the complexity introduced by the model.

A good starting point for a fitness-independent evolvability measure is the qualitative definition given by Wagner [1], which emphasizes two aspects. The first aspect of that definition can be fulfilled automatically in all evolutionary algorithms, where all individuals show heritable genetic variations and the simulated selection process can change these properties. The second aspect of Wagner's evolvability definition provides a hint of measuring evolvability: an evolvable system has to be able to acquire novel functions through genetic changes that may help the organism to survive and reproduce. The question is, what is novel functionality for the computational developmental model, and how to quantify the degree of novelty?

Based on Wagner's definition, we hypothesize that evolvability should be an indicator that reflects the relative change in the phenotype given a change in genotype. To this end, we need to define a distance measure in both genotype and phenotype, respectively, for a given genetic variation.

\section{A. Genotype Distance}

Next neighbors in the genotype space are defined by the genetic operators employed to generate offspring individuals. The easiest way to measure the distance between two genotypes is to consider both genomes as vectors with continuous values and calculate the Euclidean distance between the two vectors. However, this is nontrivial when the length and structure of two genomes are different. This is the case in our evolutionary developmental system, where not only Gaussian mutations, but also gene duplication, deletion and transposition are employed. A method for calculating the Euclidean distance between two genomes of different structures and lengths was suggested in [9], [10]. The basic idea is similar to pairwise gene sequence alignment, where the number of matches is maximized and the number of gaps (mismatches) is minimized. The gaps between the two genomes are supposed to be a result of genetic variations. Then, dynamic programming is used to find out the genetic variations that minimize a cost function. The genetic variations necessary to change from one genome to the other is then treated as the genotype distance between the two genomes. Refer to [10] for the details on how to calculate the genotype distance between two genomes of different lengths.

\section{B. Phenotype Distance}

For our developmental model for cellular growth, more than one system property can be chosen as the phenotype of the system. For instance, the morphology (shape) of the cellular structure when the growing process is complete can be seen as the phenotype. Another option is the cellular behaviors resulting from the given genome, such as cell division and cell death.

Since this work attempts to propose a general evolvability measure for developmental systems, we decided to use the dynamics of GRN as the phenotype, which we believe is generic and independent of a specific fitness function. Again, two different properties related to GRN dynamics can be considered as the phenotype of the developmental model, namely, the activity of the genes and the gradients of the TF concentrations. We found that the concentrations of transcription factors are more reliable for measuring phenotype distance because the TFs play the fundamental role in regulating the cellular behaviors.

The calculation of the phenotype distance is not straightforward even after we choose the TF concentrations as the phenotype of the developmental model for cellular growth. Again more than one possibility can be considered for calculating the phenotype distance.

- Compare the state-space trajectories of the corresponding TFs. In other words, calculate the Euclidean distance between two time series vectors of the TFs.

- Compare the steady state of the corresponding TFs of the two genomes. If the TF concentrations show oscillations, 
compare the two limit cycles in terms of the frequency, amplitude and their mean values.

- Determine the gradient field of state space that describes the dynamical system of both phenotypes. Calculate the difference of the gradient fields of both phenotypes at a number of sample points in the state space. Average over all different vectors by applying for example the Euclidean norm.

The first choice seems very easy, but it is only available for comparing TF dynamics in one cell and therefore at only one point in the environment. If there are more than one cell or the number of cells of two developmental process differs, which is almost always true in cellular growth, it is hardly possible to calculate the phenotype distance. Thus, this phenotype distance is not applicable to developmental models for multi-cellular growth.

The second possibility is very interesting to investigate, since the final morphology of the cellular structure when the growing process is complete is largely determined by the steady state of the GRN. We found however that detecting the steady state automatically, particularly detecting limit cycles in a high-dimensional space is almost intractable.

It turned out that the above-mentioned difficulties in calculating the phenotype distance for multi-cellular developmental models no longer exist in the third option. The gradient field of a TF describes the dynamics of the gene regulatory network encoded in the genome and is independent of space variables (position in the environment). It depends exclusively on the current state of all TFs and defines in which direction the state will move toward in the next time step. Therefore, we adopted this method for calculating the phenotype distance in this work. The method is very generic and applicable to all cell growth models controlled by a GRN described by differential equations.

Since we want to compare the gradient fields in the whole state space, it is meaningful to discretize the state variable (denoting the TF concentration) on equally distributed points. In this work the number of sample points for each state is chosen as $n_{\text {sample }}=5$. This means that the whole gradient field will have $n=n_{\text {sample }}{ }^{\left(2 \cdot n_{\mathrm{TF}}\right)}$ points $\left(2 \cdot n_{\mathrm{TF}}\right.$ because of external and internal TFs) to compare. A remaining question is how to determine the range of the sample points for each TF. We decided to consider a bounding box that includes the trajectories of the growing process of both phenotypes in the state space. If there is more than one cell in the individuals the bounding box could be set to cover all trajectories of all cells of both phenotypes. The upper and lower bounds of the bounding box can be found by checking the minimal and maximal values that have ever occurred during development.

The algorithm that calculates the phenotype distance by comparing the gradient fields of the TFs in the developmental model can be summarized in the following five steps:

- Find the corresponding TFs of both phenotypes by comparing their TF time series in the growing process.

- Get the sample range of all TFs by finding the maximal and minimal values of their concentrations in all cells of both phenotypes during the whole growing process.
- Divide this range into $n_{\text {sample }}$ equal intervals for every TF and combine all these samples as the discretization points for comparison.

- Calculate the gradient at all sample points for the two phenotypes, which are exclusively determined by the two genomes.

- Subtract the two gradient fields. The resulting difference vectors are then averaged over the different sample points using the Euclidean norm. The averaged vector is the phenotype distance. Each value depicts the phenotype difference of one TF.

This above phenotype distance is fitness independent and describes primarily the difference in dynamics of two genomes. It is also independent of the duration of the growing process and of the physical interactions between cells. The measure indicates the difference in the capability of genomes to show a specific dynamical behavior. As the GRN dynamics control the whole development process of the cellular system, this measure is very general yet able to describe the fundamental cellular behaviors of the genome.

\section{The Proposed Evolvability Measure}

Having defined the genotype and phenotype distances, we are finally in a position to propose a fitness-independent evolvability measure. To this end, we will introduce a statistical measure for evolvability based on a large number of next neighbors obtained by mutation (termed as mutants hereafter). The phenotype and genotype distances of all the mutants to the original individual are calculated and then plotted in a diagram to illustrate the distribution of the phenotype distance over the genotype distance, which is termed as evolvability graph. See Fig. 2 for evolvability graphs of very robust, evolvable (both robust and innovative) and highly innovative individuals.
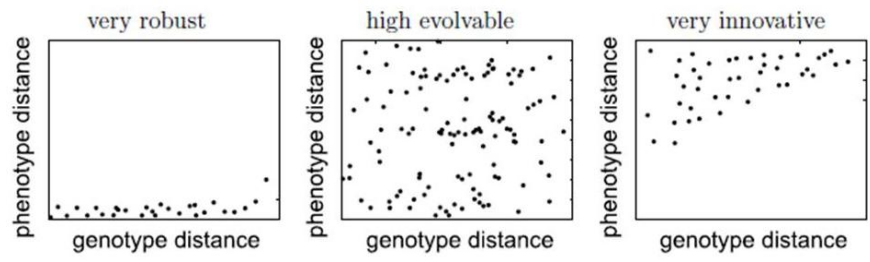

Fig. 2. Examples of a (a) very robust individual, (b) evolvable individual (both robust and innovative) and (c) a highly innovative individual.

Most properties mentioned in Section I can be found in the evolvability graph. For instance, Fig. 2 (a) shows an example of a robust individual, as most of its mutants are located in the lower part in the evolvability graph, where both small and large genotype changes can result in only small changes in the phenotype. In contrast, most of the mutants of the individual in Fig. 2 (c) are located in the upper-left part in the evolvability graph, where both small and large changes in the genotype can result in large changes in the phenotype. Therefore, this individual can be regarded to be very innovative. Finally, Fig. 2 (b) gives an example whose mutants are located quite uniformly in the evolvability graph. In this case, we consider that the individual is highly evolvable since both robust and innovative offspring can be generated from the individual. 
From the above discussions, we know that the evolvability of an individual cannot be determined by simply calculating the ratio between the phenotype distance and genotype distance. To denote the evolvability of an individual more accurately, it is necessary to compare the diversity of its mutants in the evolvability graph with a quantitative measure. In this work, we adopted the interquartile range of the fivenumber summary from the descriptive statistics [11]. The fivenumber summary is often visualized in a common box-andwhisker plot. The interquartile range (IQR) is the distance between the first and third quartiles, which is visualized as a box in the box-and-whisker plot. The first quartile is a line under which $25 \%$ of all points are arranged. The third quartile is then the line under which $75 \%$ of all data points lie. This means that in the IQR $50 \%$ of all data points are situated. The final evolvability measure $E$ can be defined as the multiplication of the IQR of the phenotype distances $\left(\mathrm{IQR}_{p}\right)$ and the IQR of the genotype distances $\left(\mathrm{IQR}_{g}\right)$ :

$$
E=\mathrm{IQR}_{p} \cdot \mathrm{IQR}_{g} .
$$

This multiplication represents an area in which an individual's mutant can be distributed upon a mutation, see Fig. 3). As mentioned above, one graph is only for one transcription factor. So the evolvability measure should be a vector of the same size as the phenotype distance vector. The mean of this vector can be used as a scalar index for measuring evolvability.

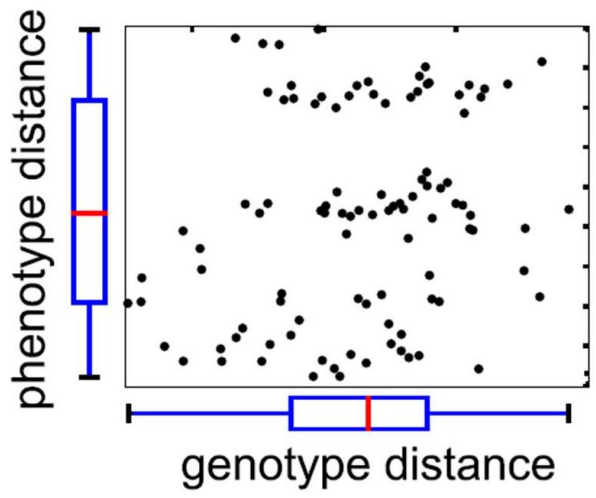

Fig. 3. Evolvability with box-and-whisker plot of all genotype and phenotype distances.

\section{EMPIRICAL STUDIES}

In this section, we illustrate how to evaluate the evolvability of a given individual. In addition, we examine, with the proposed evolvability measure, how evolvability of a population changes in evolving limit cycles. Interestingly, we show that in evolving limit circles, where evolvability is not under selection pressure, evolvability can also evolve, which confirms the findings in previous research [5], [12], [13].

\section{A. Experimental Setups}

In the simulation, the developmental model is evolved using the evolutionary algorithm described in Section II-D to achieve limit cycle. In the simulations, the parent population size is 40 and offspring population size is 100 . The fitness function is to compare the time series of the TF concentrations with a sine function, which defines the desired frequency of the limit cycle, similar to [7]. Since only the frequency matters and the amplitude is less significant in evolving limit cycles, we normalize the TF concentrations to be zero-mean and between -1 and 1:

$$
\begin{aligned}
X_{k}^{*} & =\frac{1}{X_{\max }} \cdot\left(X_{k}-X_{\text {mean }}\right), \\
S_{k} & =\sin \left(6 \pi \frac{k}{N}\right) .
\end{aligned}
$$

Finally, we use the following mean squared error as the fitness function:

$$
E=\frac{1}{N} \cdot \sum_{k=0}^{N-1}\left(X_{k}^{*}-S_{k}\right)^{2}-1.0 .
$$

Thus, the minimal fitness value is -1 .

The Runge-Kutta method has been used for solving the differential equations of the gene regulatory network, in which the step-size is set to 0.25 . The developmental time lasts for 300 iterations for each individual. In other words, $N=300$ in Equation (13).

The evolution was run for 500 generations, and 50 independent runs were performed for each setup. We report here three sets of evolutionary runs for comparison. In the first set of simulations, we limited the maximum number of RUs to six and the maximum number of SUs to three. In this setup, only mutations and gene transcriptions are used for genetic variations. That is to say, the genome length will be constant in these evolutionary runs. As indicated in [14], GRNs with this complexity should be adequate for generating sustained oscillation. No elitism strategy was adopted in the first set of evolutionary runs. In the second set of runs, all the parameter setups are the same as in the first set, and the only difference is that three elitists are always passed to the parent population of the next generation. In the third set of runs, gene duplication and deletion are also used for genetic variations in addition to mutations and gene transpositions. However, the allowed maximum number of RUs and SUs is limited to 20 to reduce the total amount of simulation time.

The best fitness in every 20 generations of 50 runs for the three setups are presented in Fig. 4(a), (b) and (c), respectively. We can see that the elitism strategy greatly improved the convergence of the evolution of limit cycles, as also found in [14]. However, the use of more RUs and SUs did not help improve the performance much, although the best individual from the runs with a variable genome length does have a better fitness than that in the runs with a fixed genome length. The dynamics of the best individuals from the three runs is presented in Fig. 5 (a), (b) and (c), respectively.

\section{B. State Space Analysis of TF Trajectories}

As discussed in Section III, we use the gradient fields of the TFs as the phenotype for investigating the evolvability of the developmental model. At first, we examine the state space trajectories of the best individuals found in the evolution (with three SUs and six RUs). We are interested in visualizing the gradient fields of the TFs in the whole space since we use the 


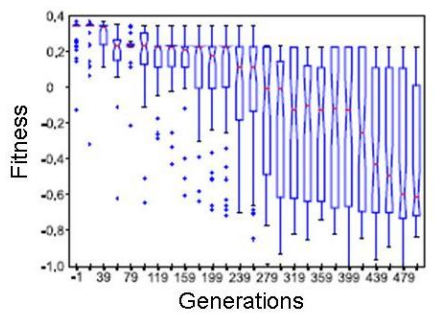

(a)

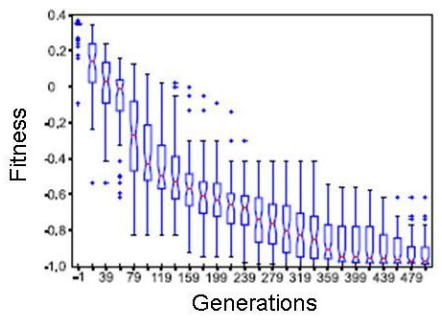

(b)

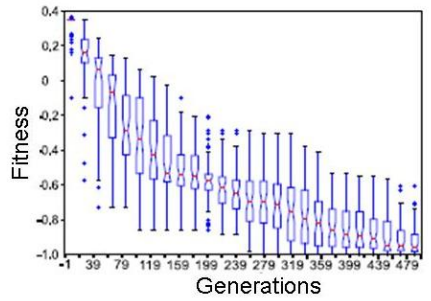

(c)

Fig. 4. Box plots of the best fitness from 50 independent runs. (a) No elitism, fixed genome length, (b) Elitism, fixed genome length, and (c) Elitism, variable genome length.

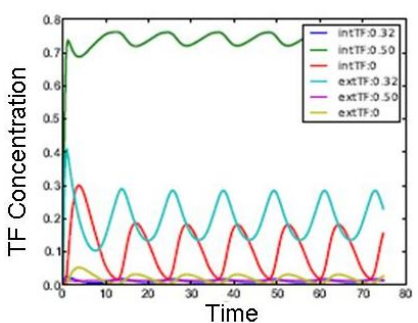

(a)

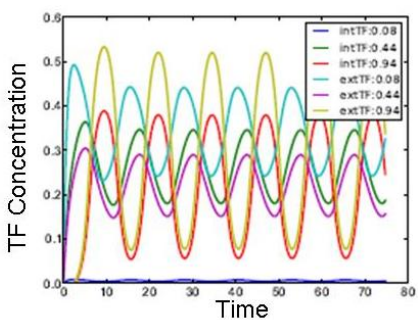

(b)

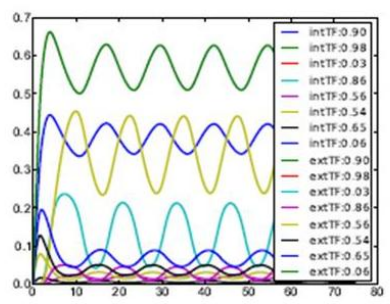

(c)
Fig. 5. Evolved TF dynamics of the best individual from the 50 independent runs. (a) No elitism, fixed genome length, (b) Elitism, fixed genome length, and (c) Elitism, variable genome length.

gradient fields for calculating the evolvability. An easy way to get the gradient field is to change the initial states of the internal TFs and let the individual to develop from all these initial states. The initial values of the external TFs were set constantly to zero. All the resulting trajectories would then give us a clue about the gradient field of the GRN dynamics. The gradient field of the above-mentioned best individual is plotted in Fig. 6. Every point (little colored dots) is the state of all internal TFs at one growing (developmental) timestep. Points in the same color belong to the same trajectory independent of the initial states. All the trajectories converge finally to the same limit cycle. The black arrows represent the gradients at each growing time-step.

In Fig. 7 the trajectories of the external TFs are also shown for the same initial states in Fig. 6. Of course, all the

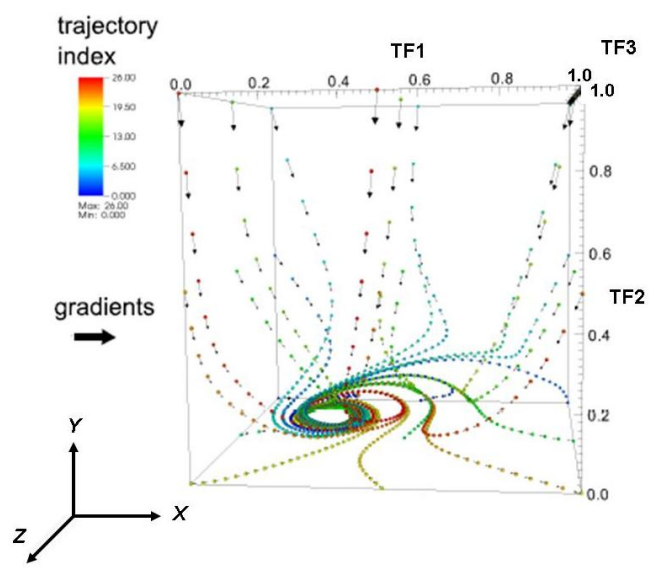

Fig. 6. State-space trajectories of three internal TFs with different initial states.

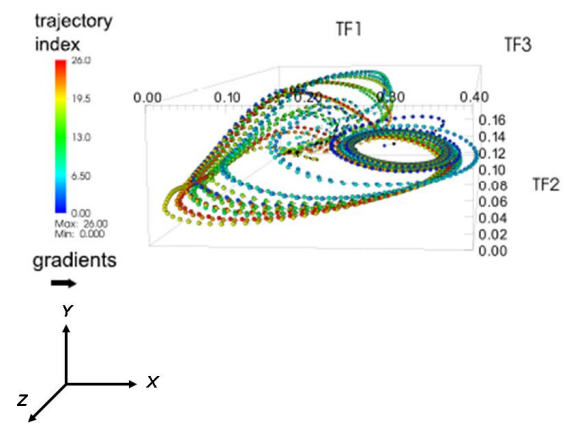

Fig. 7. State-space trajectories of three external TFs for different initial states.

trajectories start in the origin since the external TFs are always initialized to zero. Therefore, the different trajectories of the external TFs are produced from the different initial states of the internal TFs. Similar to the internal TFs, all trajectories of the external TFs end up in the same limit cycle.

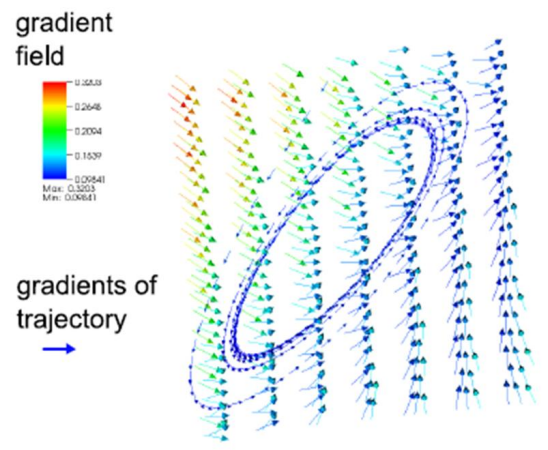

Fig. 8. Comparison of the gradient field of the sampled state space and the real trajectory of the growth process.

The phenotype distance defined in Section III-B depends on the gradients at different sampling points in the state space. By choosing specific initial states most of the trajectories take similar paths. Hence, the gradient field in a large part of the 
state-space has not been shown in Figs. 6 and 7. However, it is of interest to visualize the whole state space as it is used in the phenotype distance. The gradient is described by Equations (8) and (9). It depends only on the current state of internal and external TF concentrations. Hence, it is easy to determine the gradient vector (time derivative of the state vector) at each sample point. The resulting "flow field" of the state space is shown in Fig. 8. In the figure, the large arrows denote the gradient vectors at the sampled point. For comparison, the trajectory of the growing process is also shown denoted by small blue arrows, which is a limit cycle. Note that the sampled gradient field does not agree with the trajectory at the first sight. This is due to the dimensionality of the state space. In the chosen genome there are three concentration values for internal TFs inside the cell and three values for external TFs evaluated at cell center. The state space is therefore sixdimensional. In the visualization only three dimensions can be shown and the concentration of the external TFs change throughout the development. If for example the three internal TFs are varied, the concentration of the three external TFs cannot be determined. In this example all external TFs are set to zero to determine the gradient field. However, the result is therefore only a projection of the whole state space. Thus, it is not possible to show a limit cycle in terms of the gradient field.

\section{Evolution of Evolvability}

To investigate how evolvability of the system changes during the evolution, we need to show the evolvability graphs of the TFs showing the relationship between the genotype distances and phenotype distances. Here we show the evolvability graphs of two individuals, one is a randomly initialized with three SUs and six RUs (generation 1), and the other is the best individual obtained in generation 284 from the evolutionary runs with a fixed genome, whose TF dynamics has been studied above. The evolvability graphs of the three internal and three external TFs of the two individuals are shown in Fig. 9 and Fig. 10, respectively. In the graphs, one dot represents one mutant from the respective individual with a mutation step-size of 0.1. Comparing Fig. 9 with Fig. 10, we can observe that the spread in phenotype of the mutants in generation 284 is much larger than that of the initial individual with respect to a similar spread in genotype distance. The same conclusion can be drawn from the evolvability graphs for a step-size of 0.001 , as shown in Fig. 11 and Fig. 12, respectively.

To check the change of evolvability more quantitatively, we show the evolvability graphs of TF 3 of the two individuals in Fig. 13 again with the box plot. From the figure, the increase in phenotype distance can be observed clearly given the same genotype distance. The evolvability measure for the two individuals calculated from TF 3 is $3.91 \times 10^{-5}$ and $1.04 \times 10^{-3}$, respectively.

This finding is very interesting, since it may suggest that evolution of evolvability has been observed without an explicit selection pressure on evolvability. To further verify this hypothesis, the evolvability measures of all TFs in generation $1,100,200$ and 284 for different mutation step-sizes are
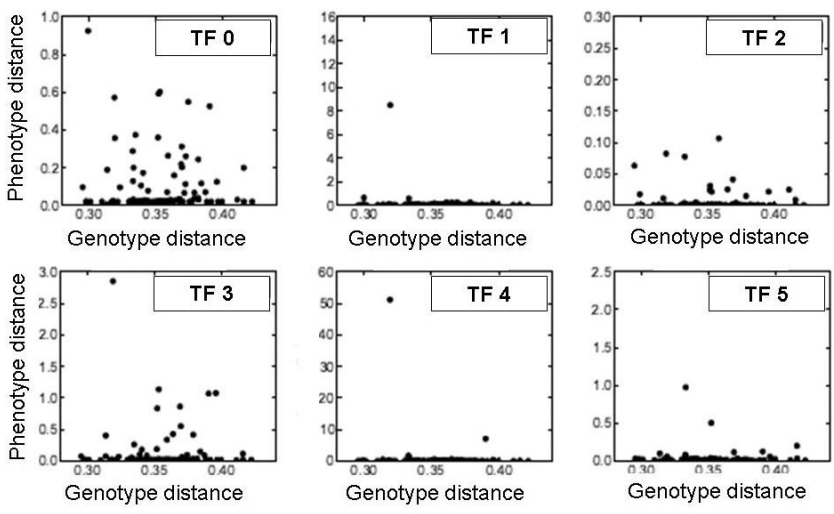

(a) Generation 1

Fig. 9. The evolvability graph of the six TFs of the individual from generation 1 , step-size $\sigma=0.1$.
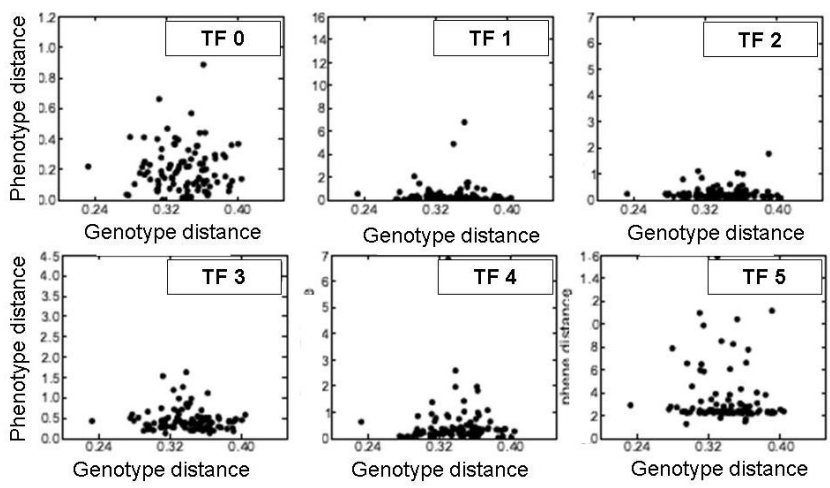

(b) Generation 284

Fig. 10. The evolvability graph of the six TFs of the best individual in generation 284, step-size $\sigma=0.1$.
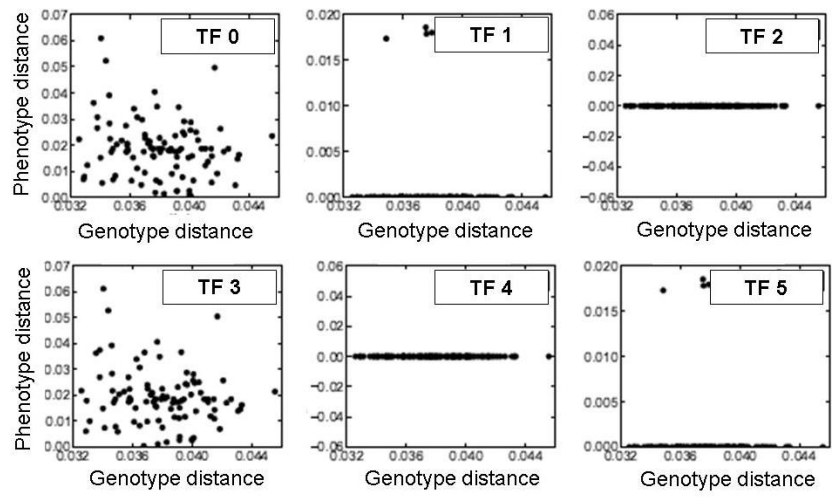

(a) Generation 1

Fig. 11. The evolvability graph of the six TFs of the individual in generation 1 , step-size $\sigma=0.001$.

calculated and plotted in Fig. 14. Note that the evolvability measure is normalized with the used mutation step-size to make them comparable. Although there are a few exceptions, a general trend of increasing evolvability over the generations can be observed for all these setups.

\section{CONCLUSIONS AND OUTLOOK}

This work suggested a new evolvability measure that is independent of a specific fitness function. This evolvability 

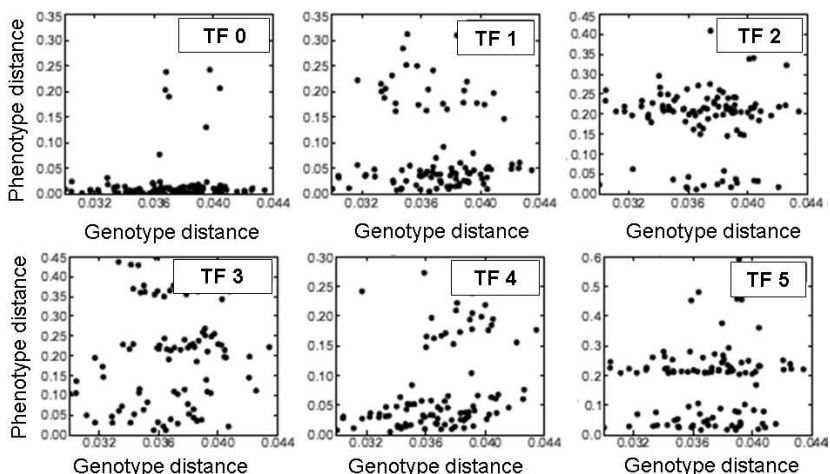

(b) Generation 284

Fig. 12. The evolvability graph of the six TFs of the best individual of generation 284, step-size $\sigma=0.001$.

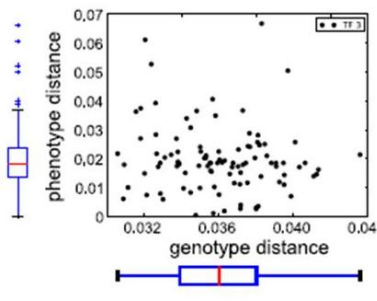

(a) Generation 1

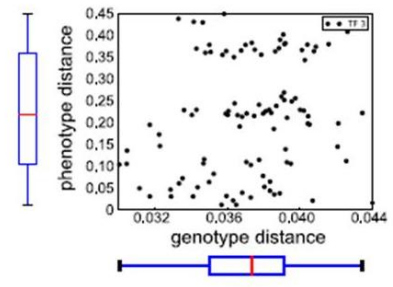

(b) Generation 284
Fig. 13. Comparison of evolvability graph of the TF 3 at generation 1 and generation 284 .

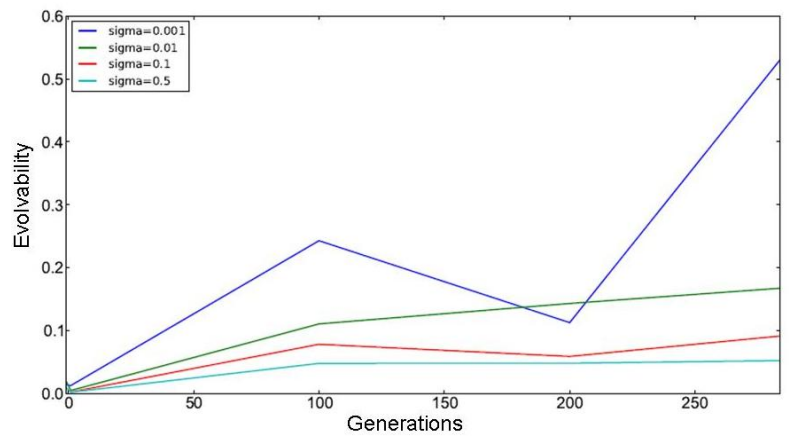

Fig. 14. Evolution of evolvability for different mutation step sizes. The evolvability measure is normalized with the mutation step-size for them to be comparable.

measure is generic in the sense that it is applicable to all developmental models in which a set of differential equations are used for modeling the gene regulatory network underlying the individual development. New methods for calculating genotype and phenotype distances were suggested. Based on the ratios between these distances calculated for a large number of mutants of an individual, the multiplication of interquartile range (IQR) in both genotype and phenotype distances is used as the evolvability measure of the individual. The IQR-based measure can take into account both individual's innovation ability and robustness, both of which are most important for evolvability. With the help of this evolvability measure, we found that evolutionary developmental systems that evolve for limit cycles have also increased their evolvability steadily, which suggests that evolution of evolvability is selectable even without an explicit selection pressure.

One weakness of the IQR-based evolvability measure is that it cannot distinguish between the case in which the mutants are uniformly distributed on the diagonal of the graph and the case in which the mutants are uniformly distributed in the whole graph. In the former case, neutrality may still be insufficient as robustness is an important factors for evolvability. We are currently considering other measures such as the Simpson's index [15] based on the genotype and phenotype distances defined in this work.

Future work also includes the investigation of change of evolvability in a statistically more significant manner. For example, we have checked the evovability of the best individual only in four different generations. It may be desirable to examine the average evolvability over all individuals in more generations. Finally, it is of great interest to verify the findings of this work for populations whose chromosome length is also varied using gene duplication and deletion.

\section{ACKNOWLEDGMENTS}

The authors are grateful to Till Steiner for inspiring discussions and programming assistance. Thanks also go to Bernhard Sendhoff for his support.

\section{REFERENCES}

[1] A. Wagner, "Robustness, evolvability, and neutrality," FEBS Letters, vol. 579, no. 8, pp. 1772-1778, 2005

[2] L. Barnett, "Evolutionary Search on Fitness Landscapes with Neutral Networks," Ph.D. dissertation, School of Cognitive and Computer Science, University of Sussex, Falmer, Brighton, 2003.

[3] P. Turney, "Increasing evolvability considered as a large-scale trend in evolution," in Proceedings of the 1999 Genetic and Evolutionary Computation Conference Workshop Program (GECCO-99 Workshop on Evolvability), 1999, pp. 43-46.

[4] J. Reisinger, K. Stanley, and R. Miikkulainen, "Towards an empirical measure of evolvability," in Proceedings of the 2005 Workshops on Genetic and Evolutionary Computation. ACM New York, NY, USA, 2005, pp. 257-264.

[5] E. Izquierdo and C. Fernando, "The evolution of evolvability in gene transcription networks," in Proceedings of Alife XI, 2008, pp. 265-273.

[6] S. Ciliberti, O. Martin, and A. Wagner, "Innovation and robustness in complex regulatory gene networks," Proceedings of the National Academy of Sciences, vol. 104, no. 34, pp. 13 591-13 596, 2007.

[7] Y. Jin, R. Gruna, I. Paenke, and B. Sendhoff, "Multi-objective optimization of robustness and innovation in redundant genetic representations," in IEEE Symposium on Multi-Criteria Decision-Makin. IEEE Press, 2009, pp. 38-45.

[8] T. Steiner, Y. Jin, and B. Sendhoff, "A cellular model for the evolutionary development of lightweight material with an inner structure," in Proceedings of the 10th Annual Conference on Genetic and Evolutionary Computation. ACM New York, NY, USA, 2008, pp. 851-858.

[9] M. Brenn, "Dreidimensionale Topologieoptimierung mit Hilfe eines Zellwachstummodells," Master's thesis, University of Darmstadt, 2008.

[10] T. Steiner, J. Trommler, M. Brenn, Y. Jin, and B. Sendhoff, "Global shape with morphogen gradients and motile polarized cells," in Congress on Evolutionary Computation, 2009, pp. 2225-2232.

[11] D. Hoaglin, F. Mosteller, and J. Tukey, Understanding robust and exploratory data analysis. Wiley, 1983.

[12] D. Earl and M. W. Deem, "Evolvability is a selectable trait," PNAS, vol. 101, pp. 11531-11 536, 2004.

[13] Y. Jin and Y. Meng, "Emergence of robust regulatory motifs from in silico evolution of sustained oscillation," BioSystems, 2009, submitted.

[14] Y. Jin, Y. Meng, and B. Sendhoff, "Influence of regulation logic on the easiness of evolving sustained oscillation for gene regulatory networks," in IEEE Symposium on Artificial Life. IEEE Press, 2009, pp. 61-68.

[15] A. Magurran, Ecological Diversity and Its Measurement. Princeton University Press, 1988. 